\title{
BANKROLL MANAGEMENT IN LARGE POKER TOURNAMENTS
}

\author{
Björn Lantz, PhD, Associate Professor \\ Department of Technology Management and Economics \\ Chalmers University of Technology \\ 41296 Gothenburg \\ SWEDEN \\ bjorn.lantz@chalmers.se
}

\begin{abstract}
This study focuses on bankroll management, defined as the process of determining the right fraction of the bankroll one should put at risk in a particular advantageous situation, examined in a poker tournament context. The aim of the study is to conduct a theoretical analysis of bankroll management based on the Kelly criterion in a typical large poker tournament, using the actual World Series of Poker Main Event payout table as an example of such tournaments. A main conclusion of this paper is that a longterm profitable poker player's expected return on investment in tournaments (i.e., the level of advantage) does not provide sufficient information to obtain an optimal bankroll management policy for the player. The level of advantage is obviously an important factor, but the player's strategic approach to the game, that is, if the player primarily tries to avoid finishing the tournament without a payout or if the player primarily tries to finish in the very top of the ranking, is also very important to consider.
\end{abstract}

Keywords: Bankroll management; gambling; poker tournaments; Kelly criterion; poker strategy; game theory

\section{INTRODUCTION}

Since the beginning of the poker boom in 2003, when the amateur Chris Moneymaker won the World Series of Poker Main Event after winning his seat via a \$39 satellite tournament, new players have regularly entered the poker scene, worked hard in order to master the fundamental underlying game theoretical concepts (e.g., Chen \& Ankenman, 2006; Ferguson \& Ferguson, 2003; von Neumann \& Morgenstern, 1944), and risen to become stars of the game. As Levitt and Miles (2014) recently demonstrated, poker is a game that includes significant elements of skill besides the obvious element of luck, so even if the outcome of a poker game in the short term is determined by chance to a large extent, in the long run, poker players benefit from being good at the 
game. However, being good at a game like poker is a concept with several meanings.

The basis for most theoretical analyses of decision making in poker is the concept of expected value $(\mathrm{EV})$. The idea is that if you make decisions that are on average profitable, you will show a profit in the long run when shortterm random variations have cancelled out (Chen \& Ankenman, 2006; Sklansky, 1999). Many poker players, even among the best, seem to agree more or less without reflection with the EV criterion. A poker related decision with a positive EV should always be made, right? Wrong. A positive EV is necessary but not sufficient for a decision to be economically rational in a poker context. According to Browne (1989), a player needs to possess three rather different qualities in order to achieve long-term success in a skill-based gambling game. First, it is essential to have a strong knowledge of the mechanics of the game, which in poker is synonymous with the ability to make decisions with a positive EV at the table. Second, the ability to prevent emotions from negatively affecting play is obviously important in poker. Most of us interested in poker have seen otherwise good players go on tilt after a bad beat and lose even more money. Third, and this is the focus of this paper, is bankroll management: the ability to determine the right fraction of the bankroll to put at risk in a particular advantageous situation. Risking too small a fraction means giving up some of the EV, but risking too large a fraction means an increased risk of ruin. Hence, bankroll management in its purest form is a question of balance.

While tactical decisions at the poker tournament table (i.e., the decision to check, bet, fold, call, or raise while playing a single hand) should be made in terms of maximizing the EV, strategic decisions (i.e., the decision to buy in to the tournament in the first place) must be made with respect to proper bankroll management. ${ }^{1}$ A tournament with a positive EV may very well constitute a negative expected growth rate (EGR) of the bankroll, in which case the player should not buy in. For example, assume that your current bankroll is $\$ 1,000,000$ and you are being offered to buy in for $\$ 100,000$ at a winner-takes-all poker tournament with 50 other players. You know that the other players on average are relatively bad, so you expect to win on average 1 out of 25 such tournaments. Should you accept the offer? It is easy to see that your EV is positive [100,000*0.04*50-100,000 $(1-0.04)=\$ 104,000]$, so you will on average make money by participating in this tournament. However, since the game will either sextuple your bankroll with a probability of 0.04 or otherwise reduce it to $90 \%$ of its previous level, the EGR of the bankroll is negative $[0.04 \log (6)+(1-0.04) \log (0.9)=-0.0128]$. Hence,

\footnotetext{
${ }^{1}$ In a cash game, where the chips have monetary value, the size of the bankroll is also affected by the tactical decisions, since players have the option to leave the table with their current stack of chips after any hand. Hence, bankroll management should, at least in theory, be part of every decision making process in such games.
} 
the correct strategic decision is to decline the offer to participate in the tournament, despite the positive EV.

The apparent inconsistency depends on the fact that the variability in the $\mathrm{EV}$ of the game is very high. Even though the EV of tournament participation in the example above was substantial-more than one buy-in-the standard deviation is actually close to ten buy-ins. Therefore, you lose too much too often when you do not win the tournament in relation to the amount you gain when you actually do win it, resulting in a negative EGR of the bankroll despite the positive EV of the game itself.

A typical large-scale multi-table poker tournament pays out a substantial part of the prize pool to the top finishers, especially to the winner. For example, the official World Series of Poker Main Event 2014 Payout table shows that with 6,481 entrants, the first prize would be 1,000 buy-ins, second price would be 505 buy-ins, and third prize would be 361 buy-ins. In such a high-variance event, a positive EV is not enough to make it rational for a player to buy in at $\$ 10,000$ to play if that $\$ 10,000$ constitutes a relatively large fraction of the player's bankroll.

There seem to be only a few previous empirical studies on issues specifically related to the bankroll management of poker players. Palomäki et al. (2014) and Palomäki et al. (2013) relate "tilting” (i.e., when emotions affect play negatively) to "loss chasing" (i.e., a decision to play at higher stakes than normal without bankroll management consideration, with the hope of winning back money that was previous lost). Their evidence suggests, among other things, that experienced players are less likely to tilt in relative terms per single hand, but more likely to tilt in the long run. Griffiths et al. (2010) found that bankroll management was one out of several factors that could predict financial success of an online poker player. Hopely and Nicki (2010) found that problem gambling (including bad bankroll management) among poker players was uniquely predicted by time played, dissociation, boredom proneness, impulsivity, and negative affective states like depression, anxiety, and stress. Neither of the above studies used random samples of poker players, however, so the practical value in terms of generalizability of these results is somewhat limited.

The aim of this study is to conduct a theoretical analysis of bankroll management in a typical large poker tournament, using the actual World Series of Poker Main Event 2014 payout table as a case example. The purpose is to investigate how different categories of players with a similar EV will differ in terms of how they should manage their bankroll in such a tournament, and to examine how the required bankroll for a certain player category varies with the EV. The remainder of the paper is structured as follows. In the next section, the Kelly criterion for optimizing the EGR of the bankroll is sketched briefly. Then, the World Series of Poker Main Event 2014 tournament structure is outlined. Thereafter, the bankroll management analyses are presented. The conclusions of these analyses and some directions for further research are given in the final section. 


\section{THE KELLY CRITERION}

Assume that a player will win a two-outcome game with probability $p$, that the odds of the game are $b$ to 1 (i.e., the player's bankroll will increase by $b$ units if he or she wins and decrease by 1 unit otherwise), and that the EV of the game is positive (i.e., $p(b+1)>1)$. If $x$ is the fraction of the bankroll the player bets, the EGR $g(x)$ of the bankroll is

(1) $g(x)=(1-p) \log (1-x)+p \log (1+b x)$.

The optimal EGR of the bankroll is given by

(2) $\frac{d g(x)}{d x}=\frac{1-p}{x-1}+\frac{b p}{1+b x}=0$

which simplifies to

(3) $x=(p(b+1)-1) / b$

which is the classical formula attributed to Kelly (1956). By betting this fraction (the Kelly bet), the EGR is maximized. Hence, betting more increases risk (in terms of bankroll variability) while decreasing the EGR, so such a strategy is dominated by the Kelly bet. Betting less also decreases the EGR but since it is less risky, such strategies may be rational for more conservative players to adopt. Betting less than nothing (i.e., taking the other side of the bet) or betting more than double the Kelly bet makes the EGR negative (Poundstone, 2010).

More generally, assume that the game has $m$ outcomes and that the profit for a unit wager for outcome $i$ is $b_{i}$ with the probability $p_{i}$, where at least one outcome is negative and at least one is positive. If $x$ is the fraction of the bankroll the player bets, the EGR $g(x)$ of the bankroll becomes

(4) $g(x)=\sum_{i=1}^{m} p_{i} \log \left(1+b_{i} x\right)$.

Hence,

(5) $\frac{d g(x)}{d x}=\sum_{i=1}^{m} \frac{b_{i} p_{i}}{1+b_{i} x}=0$ 
gives the maximum EGR of the bankroll, where the relevant solution is the smallest positive root. This is the general Kelly criterion for games with many outcomes (see e.g., Barnett, 2011 for a formal derivation).

\section{WORLD SERIES OF POKER MAIN EVENT}

The World Series of Poker (WSOP) is the largest and most well-known poker event in the world. It takes place every summer in Las Vegas and consists of many individual tournaments (in 2014 there were 65 different tournaments) of which the Main Event (ME) historically has been the largest in terms of the number of players as well as in terms of the prize pool. The entrance fee for the ME is $\$ 10,000$ per player, where $\$ 9,400$ goes back to the player collective through the prize pool and the remaining $\$ 600$ is kept as rake by the host casino and organizers. There are no specific qualification requirements (except that players must be 21 years or older), so anyone who pays $\$ 10,000$ can participate in this event and play against some of the best and most well-known poker players in the world. There are also many satellite tournaments online as well as live games where players can win a seat in the WSOP ME at a lower cost, as Chris Moneymaker did in 2003 when he eventually won the entire event.

As in almost all large poker tournaments, roughly the top $10 \%$ of the players in the WSOP ME finish "in-the-money" (ITM) and receive payouts, while the remaining players receive nothing. Table 1 shows an excerpt from the WSOP ME 2014 Payout table for 6,481 entrants, illustrating that even if players finishing at 666th place or better all do receive money, the top 3 finishers collectively receive over $30 \%$ of the price pool. Hence, as large poker tournaments typically do, the WSOP ME distributes the money in the prize pool in a very skewed way among the participants, thus creating a huge variance in the players' EVs. For most amateur players participating in such events, the variance is primarily seen as a positive factor as it means that they actually have a chance to win a lot of money if they get lucky, despite their (typically) negative EVs. For the professionals, on the other hand, the huge variance is a double edged sword, as it means that more often than not they will leave the tournament with no payout; but also that more amateurs, whose presence (typically) increases the professionals' EV, are attracted to it.

\section{BANKROLL MANAGEMENT ANALYSIS}

Assume that an average player in the field is equally likely to finish at any place. Then, there are two conceptual ways to have an advantage against an average player in a large poker tournament. First, one can have a higher probability to finish ITM than the average player. Second, one can have a weighted probability distribution for the different ITM places that favors the highest payouts. The first distinct type of player makes money in the long run by finishing ITM more often than the average player, but with a uniform 
probability distribution for the different ITM places. The second distinct type makes money in the long run by receiving a larger average payout than the average player when he or she does finish ITM, but with the same probability to actually finish ITM as the average player. The theoretical analyses below are based on those two archetypes. In practice, most long-term profitable poker tournament players are probably characterized by both these tendencies, but with varying weights.

Table 1: Excerpt from the WSOP ME 2014 Payout table for 6,481 entrants ${ }^{2}$

\begin{tabular}{|c|c|c|}
\hline Payout level & Place & Payout (USD) \\
\hline 1 & 1 & $10,000,000$ \\
\hline 2 & 2 & $5,047,370$ \\
\hline 3 & 3 & $3,617,693$ \\
\hline 4 & 4 & $2,712,211$ \\
\hline 5 & 5 & $2,046,886$ \\
\hline 6 & 6 & $1,555,305$ \\
\hline 7 & 7 & $1,189,436$ \\
\hline 8 & 8 & 915,478 \\
\hline 9 & 9 & 708,528 \\
\hline 10 & $10-12$ & 551,388 \\
\hline 11 & $13-15$ & 431,606 \\
\hline 12 & $16-18$ & 339,694 \\
\hline 13 & $19-27$ & 281,666 \\
\hline 14 & $28-36$ & 226,519 \\
\hline 15 & $37-45$ & 183,231 \\
\hline 16 & $46-54$ & 149,431 \\
\hline 17 & $55-63$ & 122,747 \\
\hline 18 & $64-72$ & 101,400 \\
\hline 19 & $73-81$ & 84,203 \\
\hline 20 & $82-90$ & 70,565 \\
\hline 21 & 91-99 & 59,298 \\
\hline 22 & $100-162$ & 50,403 \\
\hline 23 & $163-234$ & 42,695 \\
\hline 24 & $235-306$ & 36,765 \\
\hline 25 & $307-378$ & 31,428 \\
\hline 26 & $379-450$ & 27,277 \\
\hline 27 & $451-522$ & 23,719 \\
\hline 28 & 523-594 & 20,754 \\
\hline 29 & 595-666 & 18,382 \\
\hline No payout & $667-6481$ & 0 \\
\hline
\end{tabular}

2 The actual number of entrants in the WSOP ME 2014 was 6,683, so 6,481 is the next lower number of entrants to be found in the fictitious payout table published in advance by the event organizers. 
Equation (5) was used to obtain the optimal bankroll size in terms of the number of buy-ins (i.e., the inverse of the optimal fraction of the bankroll to bet) for a number of different levels of advantage, where the type of advantage is an increased probability to finish ITM compared with the average player. Similarly, equation (4) was used to derive the critical bankroll size, that is, the smallest possible number of buy-ins the player can have in the bankroll before the EGR becomes negative despite the positive EV, for the same different levels and type of advantage. The results of these calculations are presented in Table 2.

Table 2: Optimal and critical bankroll sizes for a player with an increased ITM probability

\begin{tabular}{|r|r|r|r|r|}
\hline $\begin{array}{c}\text { Increased } \\
\text { probability to } \\
\text { finish ITM }\end{array}$ & \$EV & $\begin{array}{c}\text { Expected } \\
\text { return on } \\
\text { investment }\end{array}$ & $\begin{array}{c}\text { Optimal } \\
\text { bankroll } \\
\text { size }\end{array}$ & $\begin{array}{c}\text { Critical } \\
\text { bankroll } \\
\text { size }\end{array}$ \\
\hline $10 \%$ & 340 & $3.4 \%$ & 7143 & 3448 \\
\hline $20 \%$ & 1280 & $12.8 \%$ & 1587 & 714 \\
\hline $30 \%$ & 2220 & $22.2 \%$ & 763 & 324 \\
\hline $40 \%$ & 3160 & $31.6 \%$ & 446 & 181 \\
\hline $50 \%$ & 4100 & $41.0 \%$ & 287 & 112 \\
\hline $60 \%$ & 5040 & $50.4 \%$ & 196 & 75 \\
\hline $70 \%$ & 5980 & $59.8 \%$ & 139 & 52 \\
\hline $80 \%$ & 6920 & $69.2 \%$ & 102 & 38 \\
\hline $90 \%$ & 7860 & $78.6 \%$ & 77 & 29 \\
\hline $100 \%$ & 8800 & $88.0 \%$ & 60 & 22 \\
\hline $120 \%$ & 10,680 & $106.8 \%$ & 38 & 14 \\
\hline $150 \%$ & 13,500 & $135.0 \%$ & 22 & 9 \\
\hline $200 \%$ & 18,200 & $182.0 \%$ & 12 & 5 \\
\hline & & & & \\
\hline
\end{tabular}

As one would expect, the size of both the optimal and the critical bankroll decreases rather rapidly with an increased advantage. The fact that the EGR will be positive for players with large bankrolls, as long as the advantage outweighs the rake, is of course trivial, but the lower limit of the bankroll size before the EGR becomes negative is rather high unless the player's advantage is large. There is a huge difference in required bankroll size between a player with a small advantage and a player with a large advantage.

Equations (4) and (5) were also used to obtain the optimal bankroll size and the critical bankroll size when the type of advantage is a weighted probability distribution for the different ITM places that favors the highest payouts. Because there are 29 different payout levels (see Table 1), the probability to finish at payout level 1 (i.e., to win the tournament) is increased by a factor $Z$ while the probability to finish at payout level 29 is reduced by 
the same absolute amount, the probability to finish at payout level 2 (i.e., to finish as runner-up) is increased by the factor $Z^{*}(13 / 14)$ while the probability to finish at payout level 28 is reduced by the same absolute amount, and so on. Hence, the probability distribution of the 29 payout levels is "tilted" linearly with payout level 15 as midpoint and the total probability to finish ITM is thus constant irrespective of the value of $Z$. A higher value of $Z$ obviously corresponds to a more apparent "tilt" of the probability distribution, and, hence, a higher $\$ E V$. Different values of $Z$ were used to obtain the same \$EV levels as in Table 2 in order to make direct comparisons possible. The resulting optimal and critical bankroll sizes are displayed in Table 3.

Table 3: Optimal and critical bankroll sizes for a player with a "tilted" payout level probability distribution

\begin{tabular}{|r|r|r|r|r|}
\hline $\begin{array}{c}\text { The weighting } \\
\text { factor } Z\end{array}$ & \multicolumn{1}{|c|}{$\$ \mathrm{EV}$} & $\begin{array}{c}\text { Expected } \\
\text { return on } \\
\text { investment }\end{array}$ & $\begin{array}{c}\text { Optimal } \\
\text { bankroll size }\end{array}$ & $\begin{array}{c}\text { Critical } \\
\text { bankroll } \\
\text { size }\end{array}$ \\
\hline 0.2427 & 340 & $3.4 \%$ & 8130 & 3937 \\
\hline 0.4855 & 1280 & $12.8 \%$ & 2041 & 935 \\
\hline 0.7282 & 2220 & $22.2 \%$ & 1136 & 498 \\
\hline 0.9709 & 3160 & $31.6 \%$ & 781 & 327 \\
\hline 1.2137 & 4100 & $41.0 \%$ & 588 & 239 \\
\hline 1.4564 & 5040 & $50.4 \%$ & 469 & 187 \\
\hline 1.6992 & 5980 & $59.8 \%$ & 389 & 152 \\
\hline 1.9419 & 6920 & $69.2 \%$ & 331 & 127 \\
\hline 2.1846 & 7860 & $78.6 \%$ & 289 & 107 \\
\hline 2.4274 & 8800 & $88.0 \%$ & 256 & 95 \\
\hline 2.9128 & 10,680 & $106.8 \%$ & 207 & 75 \\
\hline 3.6410 & 13,500 & $135.0 \%$ & 162 & 57 \\
\hline 4.8547 & 18,200 & $182.0 \%$ & 119 & 40 \\
\hline
\end{tabular}

Here, too, the sizes of both the optimal and the critical bankroll decrease with an increased advantage; and there is a large difference in required bankroll size between a player with a small advantage and a player with a large advantage. The main differences, however, are that when the type of advantage is a weighted probability distribution for the different ITM places that favors the highest payouts, the decrease in the sizes of the optimal and critical bankroll for increasing \$EV is markedly slower, and the optimal as well as critical bankroll sizes are larger for all \$EV levels, especially so for the most profitable players. 


\section{CONCLUSION}

Bankroll management can be seen as the process of determining the right fraction of their bankroll that players should put at risk in a particular advantageous situation. A fundamental bankroll management problem in the poker tournament context is that a large high stakes tournament such as the WSOP ME may, due to the high variance in the EV, represent a negative EGR of the player's bankroll, even though the EV of participation in the tournament itself is substantially positive. In order to avoid this problem, one simply cannot play at too high stakes. On the other hand, when playing at too small stakes, the EGR of the bankroll may be negligible in relation to the potential. Balancing these two forces is the actual core of bankroll management in any type of advantageous game.

A main conclusion of this paper is that a long-term profitable poker player's expected return on investment in tournaments (i.e., the level of advantage) does not provide sufficient information to obtain an optimal bankroll management policy for the player. The level of advantage is obviously an important factor, but the player's strategic approach to the game, that is, if the player primarily tries to avoid finishing the tournament without a payout (the low risk approach) or if the player primarily tries to finish in the very top of the ranking (the high risk approach), is also very important to consider. This is clearly illustrated by the fact that a WSOP ME player with a low risk approach and an expected return on investment of around $40 \%$ has about the same optimal bankroll size as a WSOP ME player with a high risk approach and an expected return on investment of around 80\%. Hence, there is definitely no bankroll size that, even as a rule of thumb, can be said to be about right for most tournament poker players. The players' skill levels and their strategic approach to the game must both be considered in their bankroll management process.

Although all long-term profitable tournament poker players are well aware of their strategic approach to the game, the skill level of a player is difficult to determine with some precision. How can you reasonably know what your expected return on investment is in a particular tournament? To avoid the hubris factor, many professionals seem to derive estimates of their advantage from previous results; that is, they use their actual average return on investment in a large number of similar tournaments as an estimate of their expected return on investment in a particular tournament. While such an approach is questionable in terms of both validity and reliability for the individual player, it seems reasonable to assume that the best poker players may have an expected return on investment of perhaps 25\% in tournaments in general, even though in the WSOP Main Event specifically it could very well be much higher, because the proportion of amateurs playing that event is very high in relation to the stakes (Pempus, 2014; Plant, 2014). As the results in this paper have shown, efficient bankroll management in poker tournaments depends fundamentally on reliable estimates of the players' actual advantages 
against the field in the tournaments they consider entering. This highlights the need for sensitivity analyses in the bankroll management process, because it is dangerous for players with a small bankroll to overvalue their $\$ E V$. The difference in assumed and actual skill level that makes an optimal EGR become a negative EGR is not that large. When in doubt, the safe way is simply to play at lower rather than at higher stakes, because the EGR can by definition never become negative with a bankroll larger than the optimal one, as long as the $\mathrm{EV}$ is positive.

As the results in this paper show, a player with an expected return on investment of around 25\% needs a bankroll size somewhere between 500 and 1,000 buy-ins to be able to play a tournament of the WSOP ME size and structure efficiently. Playing with a smaller bankroll means higher variability in the bankroll in combination with a lower EGR, something that cannot be rational in a risk-reward perspective. Playing with a larger bankroll, on the other hand, may be rational for some players because the lower risk can compensate for the lower EGR. Although professional poker players typically do not share detailed information regarding the size of their bankrolls publicly, it seems safe to assume that it is much more common that players play at too high stakes than at too low stakes from a bankroll management perspective.

There are several directions for future research in this field. First, from a theoretical perspective, the classical observation that the EGR becomes negative when the player bets more than double the Kelly bet evidently does not apply in games with many outcomes. Where the verge is seems to depend on the structure and probability distribution of the outcomes in the specific game, but in a poker tournament of the WSOP ME type, it fluctuates between double and triple the Kelly bet. Second, from an empirical perspective, we need to increase our understanding of how professional poker players (and other gamblers) manage their bankrolls. From a quantitative perspective, the amount of scientific and other literature on the mechanics of poker seems to overshadow the amount of literature on the other two qualities needed for success, that is, the ability to prevent emotions from negatively affecting play and the ability to manage bankrolls properly. This can be seen as an indication that the potential for improvement for the average ambitious professional player lies mainly with these two qualities.

Finally, it should be mentioned that a limitation of the analysis in this study is the assumption that players only play to win money and do not assign value to other things. In practice, of course, a win in a WSOP event is in itself very prestigious, especially among the most celebrated professionals who probably look at each other's bracelets ${ }^{3}$ with a combination of admiration and envy. Only a few days before these very lines were written, Phil Helmuth, one

\footnotetext{
${ }^{3}$ A bracelet is awarded to the winner of every event in the WSOP, in addition to the prize money. Such bracelets are among the most coveted non-monetary prizes poker players can win.
} 
of the most successful poker players of all time, won his fourteenth WSOP event. Although the monetary payout for the win of course was considerable, Helmuth seemed to value the win as such as substantially higher than the prize money.

\section{REFERENCES}

Barnett, T. (2011). How much to bet on video poker. Chance, 24, 10-14.

Browne, B. (1989). Going on tilt: Frequent poker players and control. Journal of Gambling Behavior, 5, 3-21.

Chen, B., \& Ankenman, J. (2006). The Mathematics of Poker. Pittsburgh: ConJelCo.

Ferguson, C., \& Ferguson, T. (2003). On the Borel and von Neumann poker models. Game Theory and Applications, 9, 17-32.

Griffiths, M., Parke, J., Wood, R., \& Rigbye, J. (2010). Online poker gambling in university students: Further findings from an online survey. International Journal of Mental Health and Addiction, 8, 82-89.

Hopely, A., \& Nicki, R. (2010). Predictive factors of excessive online poker playing. Cyberpsychology, Behaviour, and Social Networking, 13, 379-385.

Kelly, J. (1956). A new interpretation of information rate. The Bell System Technical Journal, July, 917-926.

Levitt, S., \& Miles, T. (2014). The role of skill versus luck in poker: Evidence from the World Series of Poker. Journal of Sports Economics, 15, 31-44.

Palomäki, J., Laakasuo, M., \& Salmela, M. (2013). 'This is just so unfair!': A qualitative analysis of loss-induced emotions and tilting in on-line poker. International Gambling Studies, 13, 255-270.

Palomäki, J., Laakasuo, M., \& Salmela, M. (2014). Losing more by losing it: Poker experience, sensitivity to losses and tilting severity. Journal of Gambling Studies, 30, 187-200.

Pempus, B. (2014). A Look at the Probability of What Poker Pro Mark Newhouse Just Did at the WSOP. Retrieved from http://www.cardplayer.com/poker-news/18066a-look-at-the-probability-of-what-poker-pro-mark-newhouse-just-did-at-the-wsop (accessed June 17, 2015).

Plant, D. (2014). Why You'll Never Make a Living Playing Live Poker Tournaments. Retrieved from http://regressing.deadspin.com/why-its-hard-for-poker-pros-tomake-a-living-playing-l-1526098295 (accessed June 17, 2015).

Poundstone (2010). Fortune's Formula: The Untold Story of the Scientific Betting System That Beat the Casinos and Wall Street, New York: Hill and Wang.

Sklansky, D. (1999). The Theory of Poker. Las Vegas: Two Plus Two Publishing.

Von Neumann, J., \& Morgenstern, O. (1944). Theory of Games and Economic Behavior. Princeton: Princeton University Press. 